\title{
Teaching Cases of the Month
}

\section{A 60-Year Old Man Presenting With Yellow Nail Syndrome}

\author{
Ariel Modrykamien MD and Omar Minai MD
}

\section{Introduction}

Yellow nail syndrome is a rare disorder of unclear etiology. Since its original description by Samman and White, ${ }^{1}$ many other reports and associations have been described. Current evidence suggests that its pathologic mechanism is based on lymphatic dysfunction, which is thought to be acquired rather than congenital. However, our understanding of its pathogenesis is still speculative, as it relies on anecdotal observations. We report a case of a man who presented with dry cough, shortness of breath, bilateral lower-extremity edema, and pleural effusion. Yellow nail syndrome was diagnosed.

\section{Case Summary}

A 60-year-old man presented with 2 months of persistent dry cough. He noticed dyspnea with moderate effort, such as walking 2-3 blocks, and bilateral lower-extremity edema waxing and waning over 2 months. He was a hairdresser and his history included 2 pneumonias. He denied other systemic symptoms and had no other medical or surgical history. He was a 35-pack-year smoker.

Vital signs were: blood pressure 160/100 $\mathrm{mm} \mathrm{Hg}$, heart rate 111 beats/min, respiratory rate 20 breaths $/ \mathrm{min}$, temperature $36.9^{\circ} \mathrm{C}$, oxygen saturation $96 \%$ on room air. He had decreased breath sounds in the left base, symmetric bilateral lower-extremity non-pitting edema, and yellow fingernails. His hematocrit was $48 \%$. He had normal white blood cell count, platelet count, coagulation studies, liver, and kidney function tests, blood glucose, and urinalysis.

Dr Modrykamien is affiliated with the Pulmonary, Critical Care, and Sleep Medicine Division, Creighton University Medical Center. Omaha, Nebraska. Dr Minai is affiliated with the Respiratory Institute, Cleveland Clinic, Cleveland, Ohio.

The authors have disclosed no conflicts of interest.

Correspondence: Ariel Modrykamien MD, Pulmonary, Critical Care, and Sleep Medicine Division, Creighton University Medical Center, 601 N 30th Street, Suite 3820, Omaha NE 68131. E-mail: arielmodrykamien@creighton.edu.

DOI: $10.4187 /$ respcare.00942
Blood total protein was $5.2 \mathrm{~g} / \mathrm{dL}$, and lactate dehydrogenase was $350 \mathrm{U} / \mathrm{L}$. His $\mathrm{FEV}_{1}$ was $64 \%$ of predicted, forced vital capacity was $60 \%$ of predicted, the ratio of $\mathrm{FEV}_{1}$ to forced vital capacity was 0.81 , and his diffusion capacity for carbon monoxide was $97 \%$ of predicted.

Echocardiogram revealed normal cavities and valve function. Left-side thoracentesis obtained a cloudy pleural fluid with total protein $3.9 \mathrm{~g} / \mathrm{dL}$, lactate dehydrogenase $144 \mathrm{U} / \mathrm{L}$, glucose $100 \mathrm{mg} / \mathrm{dL}$, and pH 7.6, with 79\% lymphocytes. Pleural fluid cultures for bacterial, fungal, and acid-fast bacilli were negative. Cytology was negative for malignant cells. Flow cytometry was negative for chronic lymphoproliferative disorders. Tuberculosis skin test was also negative.

\section{Radiology Findings}

Chest radiograph revealed a left-side pleural effusion (Fig. 1). High-resolution computed tomogram showed a left-side pleural effusion with areas of loculation (Fig. 2). Lymphoscintigraphy of the lower extremities (Fig. 3) revealed a decreased number of lymph channels in the left leg and the lateral aspect of the right leg, and poor migration of the tracer cephalad.

He underwent video-assisted thoracoscopic surgery with pleural biopsy as part of the work up for lymphocytic exudate. The biopsy revealed collagenous tissue with few chronic inflammatory cells. He was diagnosed yellow nail syndrome and underwent talc pleurodesis. Other recommendations were bandage wraps and exercises for his lower-extremity lymphedema. After 2 months his respiratory symptoms subsided and his lymphedema improved.

\section{Discussion}

Yellow nail syndrome was described in 1964 by Samman and White, ${ }^{1}$ who reported 13 patients with slowgrowing nails associated with lymphedema, and hypothesized that anatomical and/or functional abnormality of the lymphatic vessels was the principal cause. While lymphedema is the most common finding, other manifestations include bronchiectasis, sinusitis, recurrent pneumonia, and pleural effusions. Classically, yellow nail syn- 


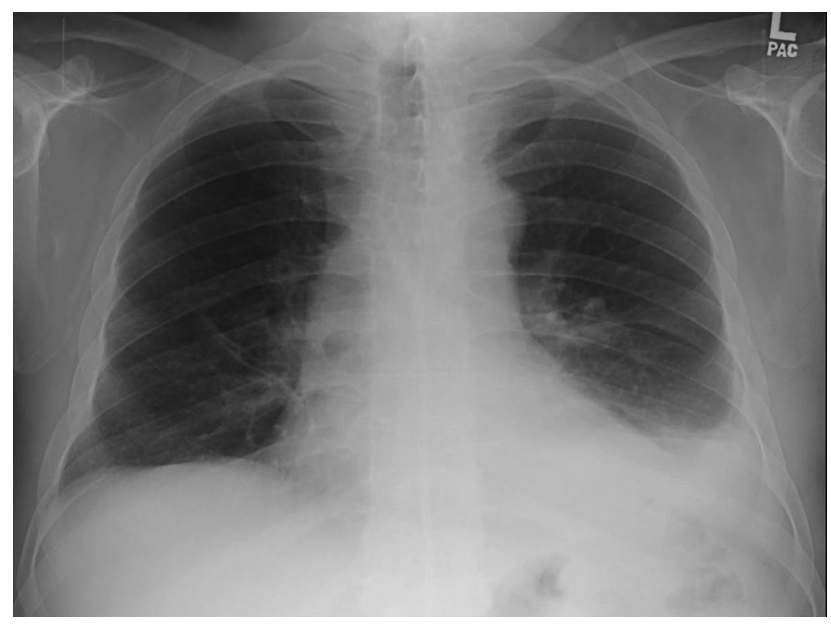

Fig. 1. Radiograph shows left-side pleural effusion.

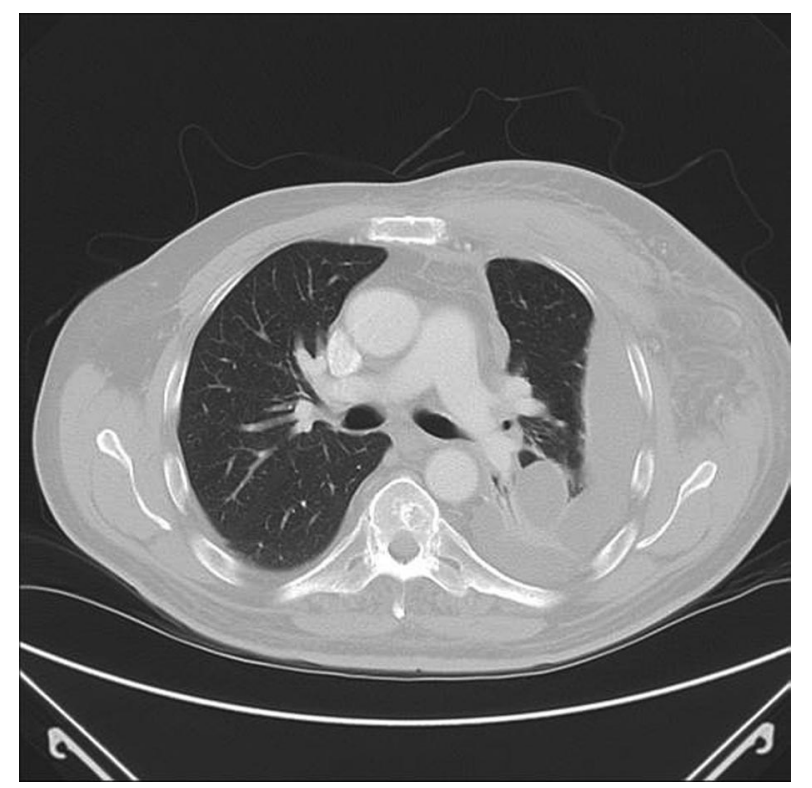

Fig. 2. Computed tomogram shows loculated left-side pleural effusion.

drome is described as a triad of yellow nails, lymphedema, and respiratory tract involvement: usually pleural effusion. ${ }^{2}$ The diagnosis of yellow nail syndrome requires 2 of these 3 findings. One third of patients present with yellow nails. Other nail abnormalities include overcurvature, thickening, onycholysis, and loss of lunulae and cuticles. In a retrospective review of 41 consecutive patients with yellow nail syndrome, the median age at diagnosis was 61 years. ${ }^{3}$ Sixty-three percent of the patients had lymphedema, $56 \%$ had chronic cough, $46 \%$ had pleural effusions, $44 \%$ had bronchiectasis, and 9\% had recurrent pneumonias. The pleural effusion associated with yellow nail syndrome is exudative and usually bilateral. However, the pleural fluid lactate dehydrogenase level is low, with a lactate dehydrogenase ratio in the

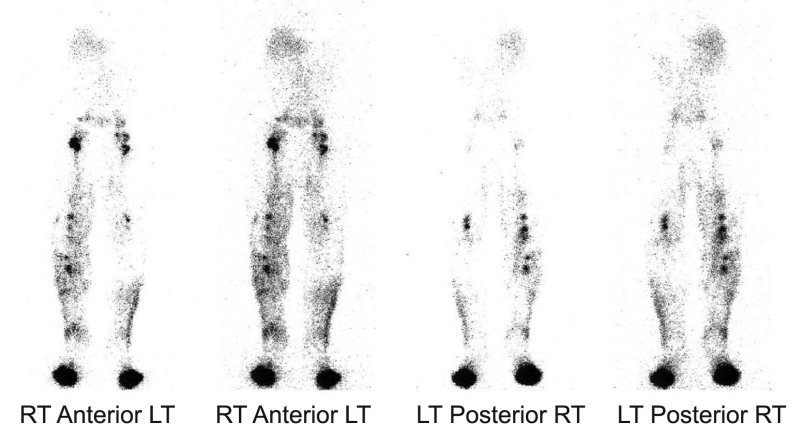

Fig. 3. Lymphoscintigraphy shows a decreased number of lymph channels in the left leg and the lateral aspect of the right leg.

transudative range. Pleural cell count remains below 2,000 cells $/ \mu \mathrm{L}$, with lymphocyte predominance $(>60-80 \%)$. These pleural effusions tend to recur. ${ }^{4}$

It was initially thought that yellow nail syndrome was a hereditary disorder, transmitted in an autosomal dominant fashion. However, in a case series of 11 patients with yellow nail syndrome, only one had a positive family history, which suggests that yellow nail syndrome is probably not a primary genetic disease. ${ }^{5}$ It was also speculated that lymphatic blockage might not be enough to explain all the manifestations of this syndrome. Other theories suggested that increased microvascular permeability at sites such as the pleura, liver, extremities, and intestine, contribute to the pathogenesis of this syndrome, ${ }^{6}$ and others have suggested hypoplasia of lymphatic channels. ${ }^{7}$

Once the diagnosis of yellow nail syndrome is made, treatment is symptomatic. Local steroid injection and oral vitamin E (800 UI daily for up to 18 months) have been reported to be successful in treating the yellow nails. Topical vitamin $\mathrm{E}$ ( $5 \%$ solution, 2 drops twice daily) has been successful as well. Its property as a potent free radical scavenger might be responsible for this benefit. ${ }^{8}$ Optimal treatment of bronchiectasis involves pulmonary hygiene, with postural drainage, Flutter valve, chest physiotherapy, or high-frequency chest wall compression (eg, the Vest, Hill-Rom, St Paul, Minnesota). Lymphedema is managed with bandage wraps, exercise, and external pneumatic compression. Pleural effusions are treated with pleurodesis, pleuroperitoneal shunt, and pleurovenous shunt. Chemical pleurodesis with talc, tetracycline, and quinacrine has also been successful in controlling effusions. ${ }^{9}$

\section{Teaching Points}

- Yellow nail syndrome is a triad of yellow nails, lymphedema, and pulmonary involvement. Two of these 3 findings are required to make the diagnosis. 


\section{A 60-Year Old Man Presenting With Yellow Nail Syndrome}

- The pleural effusions are exudates with a high total protein concentration, low lactate dehydrogenase level, and are predominantly lymphocytic.

- There is no specific treatment for yellow nail syndrome; the goal is symptom control.

\section{REFERENCES}

1. Samman PD, White WF. The "yellow nail" syndrome. Br J Dermatol 1964;76:153-157.

2. Alkadhi H, Wildermuth S, Russi EW, Boehm T. Yellow nail syndrome. Respiration 2005;72(2):197.

3. Maldonado F, Tazelaar HD, Wang CW, Ryu JH. Yellow nail syndrome: analysis of 41 consecutive patients. Chest 2008;134(2):375-381.
4. Maldonado F, Ryu JH. Yellow nail syndrome. Curr Opin Pulm Med 2009;15(4):371-375.

5. Hoque SR, Mansour S, Mortimer PS. Yellow nail syndrome: not a genetic disorder? Eleven new cases and a review of the literature. Br J Dermatol 2007;156(6):1230-1234.

6. D’Alessandro A, Muzi G, Monaco A, Filiberto S, Barboni A, Abbritti G. Yellow nail syndrome: does protein leakage play a role? Eur Respir J 2001;17(1):149-152.

7. Bull RH, Fenton DA, Mortimer PS. Lymphatic function in the yellow nail syndrome. Br J Dermatol 1996;134(2):307-312.

8. Williams HC, Buffham R, du Vivier A. Successful use of topical vitamin $\mathrm{E}$ solution in the treatment of nail changes in yellow nail syndrome. Arch Dermatol 1991;127(7):1023-1028.

9. Glazer M, Berkman N, Lafair JS, Kramer MR. Successful talc slurry pleurodesis in patients with nonmalignant pleural effusion. Chest 2000;117(5):1404-1409. 